\title{
LA CIRUGÍA TORÁCICA VIDEO-ASISTIDA (VATS) TEMPRANA, MEJORA LA CALIDAD DE VIDA EN LOS DERRAMES PLEURALES NEOPLÁSICOS COMPLICADOS.
}

\author{
EARLY VIDEO-ASSISTED THORACIC SURGERY (VATS) IMPROVES QUALITY OF LIFE IN \\ COMPLICATED NEOPLASTIC PLEURAL EFFUSIONS.
}

\author{
F. N. Alvarez Padilla ${ }^{1}$, E. Schiavoni, E. F'. Bustos Mario'
}

\section{Resumen:}

Introducción: El derrame pleural neoplásico (DPN) implica una enfermedad oncológica avanzada. La biopsia pleural por cirugía torácica endoscópica permite el diagnóstico en más del $90 \%$ de los casos y la instrumentación del espacio pleural complicado, mejorando los resultados de la técnica.

Material y Método: Se realizó un análisis retrospectivo de pacientes con DPN operados para la realización de una pleurodesis química con talco. Se formaron dos grupos, uno con derrame pleural neoplásico complicado (DPNC) y otro con derrame pleural neoplásico no complicado (DPNNC). En el grupo con DPNC se realizó "maniobras de liberación - expansión". Se compararon las variables entre ambos grupos para el análisis pertinente.

Resultados: Se analizaron 28 pacientes con DPN tratados con pleurodesis química por cirugía torácica endoscópica. La edad promedio fue de 62,64 años. El compromiso pleural por patología mamaria fue la forma más frecuente $(46,4 \%)$. No se hubo diferencia en cuanto a complicaciones $(p=0,31)$ y riesgo de defunción a los 30 días $(\mathrm{p}=1,09)$ con el manejo agresivo del espacio pleural. La demora en la indicación de pleurodesis se relacionó con un mayor índice de complicaciones $(p=0,002)$ y mayor probabilidad de defunción dentro de los 30 días $(p=0,008)$. La mayoría de pacientes se reinsertó a sus tareas diarias, con buena tolerancia a la disnea luego del procedimiento.

Conclusión: En los pacientes con DPNC, las "maniobras de liberación - expansión pulmonar" descriptas, aumentarían las chances de mejorar los resultados con bajo riesgo. La pleurodesis química temprana mejora la calidad de vida de los pacientes portadores de un DPN.

Palabras clave: derrame pleural maligno; toracoscopía; cirugía asistida por video.

\section{Abstract:}

Introduction: Malignant pleural effusion (MPE) involves advanced cancer disease. Pleural biopsy for endoscopic thoracic surgery allows diagnosis in more than $90 \%$ of cases and instrumentation of the pleural space, improving the results of the technique.

Material and Method: We performed a retrospective analysis of patients with MPE who underwent a talc chemical pleurodesis. Two groups were formed, one with complicated malignant pleural effusion (CMPE) and another with uncomplicated malignant pleural effusion (NCMPE). In the group with CMPE, "release expansion maneuvers" were performed. The variables between the two groups were compared for the relevant analysis.

Results: We analyzed 28 patients with MPE treated with chemical pleurodesis by endoscopic thoracic surgery. The average age was 62.64 years. Pleural involvement due to breast disease was the most frequent form (46.4\%). There was no difference between complication rate $(p=0.31)$ and the risk of death at 30 days $(p=1.09)$ with aggressive management of pleural space. The delay pleurodesis indication was related to a higher rate of complications $(p=0.002)$ and a higher probability of death within 30 days $(p=$ 0.008). The majority of patients return to their daily tasks, with good tolerance to dyspnea following the procedure.

Conclusion: In patients with complicated MPE, the "lung-release maneuvers" described above would increase the chances of improving outcomes at low risk. Early chemical pleurodesis improves the quality of life of patients with PND.

Key Words: pleural effusion; malignant, thoracoscopy; video-assisted surgery.

\footnotetext{
${ }^{1}$ Servicio de Cirugía Torácica. Hospital Privado. Centro Médico de Córdoba. Córdoba. Argentina.
} 


\section{Introducción}

El derrame pleural neoplásico (DPN) es la expresión de una enfermedad oncológica avanzada, con una sobrevida estimada en pocos meses en la mayoría de los casos ${ }^{[1]}$. Las neoplasias de mama, pulmón y los linfomas, son sus causas más frecuentes. Otras estirpes tumorales que pueden desarrollar DPN son: los tumores de ovario, del tracto gastrointestinal y el mesotelioma pleural. Siempre que sea posible, debería realizarse la confirmación histológica mediante biopsia pleural para no diferir el tratamiento oncológico. En éste sentido, la biopsia pleural por videotoracoscopía (VTC) o cirugía torácica video asistida (VATS) permite el diagnóstico en más del $90 \%$ de los casos ${ }^{[1]}$. La cirugía torácica endoscópica además de confirmar el compromiso pleural, brinda la posibilidad de actuar sobre la acumulación de líquido, antes que la progresión de la enfermedad afecte la expansión pulmonar (pulmón atrapado), contraindicando un procedimiento de sellado pleural ${ }^{[2,3]}$. La VTC/VATS son procedimientos de bajo riesgo en casos seleccionados, que permiten la instrumentación de espacios pleurales con depósitos de fibrina, coágulos y/o adherencias que por otros métodos no es factible realizar, mejorando los resultados de la pleurodesis química ${ }^{[1,4,5]}$.

\section{Objetivos}

El objetivo principal fue evaluar el impacto del manejo del derrame pleural neoplásico complicado (DPNC) mediante VATS temprana y el uso de maniobras de liberación - expansión pulmonar, en el tratamiento del DPN y la calidad de vida de éstos pacientes.

Como objetivo secundario, se evaluó, si el uso de toracocentesis seriadas previo a la pleurodesis química por VATS, tiene repercusión en el manejo del DPN y sus resultados.

\section{Materiales y Métodos}

Se realizó un análisis retrospectivo, de pacientes con diagnóstico de DPN operados por videotoracoscopía para la realización de una pleurodesis química con talco entre enero/2007 y enero/2013. Se identificó aquellos casos que presentaron un DPNC, diagnosticado al momento de la cirugía. Se definió como DPNC, al derrame asociado a depósitos de fibrina, coágulos y/o adherencias en el espacio pleural, que imposibilitan la correcta expansión pulmonar. Se tuvo en cuenta los pacientes con "pulmón en vías de atrapamiento", definiéndose éste como la falla de expansión producto del engrosamiento pleural ("peel") por contacto prolongado con el líquido pleural y la acumulación de fibrina en la superficie, pero en donde aún es posible instrumentar el espacio pleural para permitir la reexpansión pulmonar.

\section{Selección de pacientes}

Un total de 44 pacientes con DPN tratados con pleurodesis química, fueron analizados. Se descartaron seis casos por seguimiento y/o datos incompletos o por recibir tratamiento con otro agente esclerosante diferente del talco (doxiciclina). Otros nueve no se tuvieron en cuenta para el análisis, por presentar un pulmón atrapado ya instaurado sin posibilidades de reexpansión al momento de la cirugía, o porque la pleurodesis se realizó por tubo de drenaje. Un último caso, se descartó por no lograrse el diagnóstico definitivo, aun con la realización de técnicas de inmunohistoquimica. En la figura 1.se detalla el proceso de selección de los casos, con un total de 28 pacientes que finalmente fueron incluidos para la realización del trabajo.

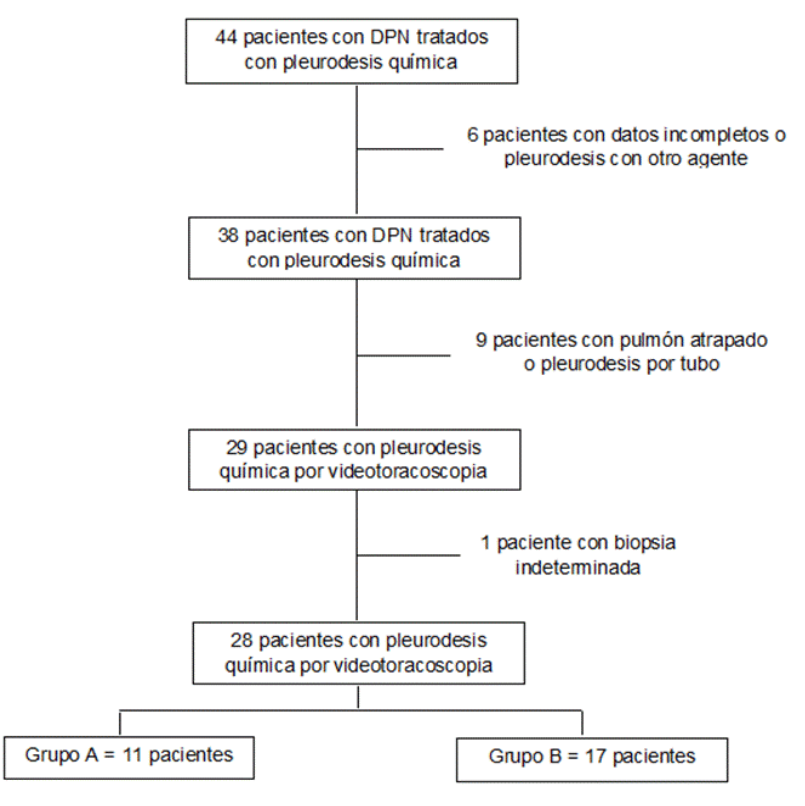

Figura 1.- Esquema de selección de pacientes

Se formaron dos grupos, uno con portadores de un derrame pleural neoplásico complicado (DPNC) y otro con aquellos con un derrame pleural neoplásico no complicado (DPNNC). En el grupo con DPNC se realizó lo que denominamos "maniobras de liberación expansión" con la finalidad de lograr la mayor expansión pulmonar posible. Éstas maniobras consisten en: 1) clampeo o infiltración del nervio frénico a nivel del ángulo cardio-frénico con $10 \mathrm{cc}$ bupivacaína al 0,5\%, 2) liberación de las adherencias diafragmáticas parietales con disección roma, 3) sección del ligamento 
pulmonar inferior, 4) "decorticación" de la base y el borde pulmonar inferior, con liberación de las cisura mayor. Figura 2.- Estas maniobras se realizaron en conjunto o por separado, según el caso, siempre y cuando el paciente a tratar tuviera un buen estado clínico al momento de la cirugía.

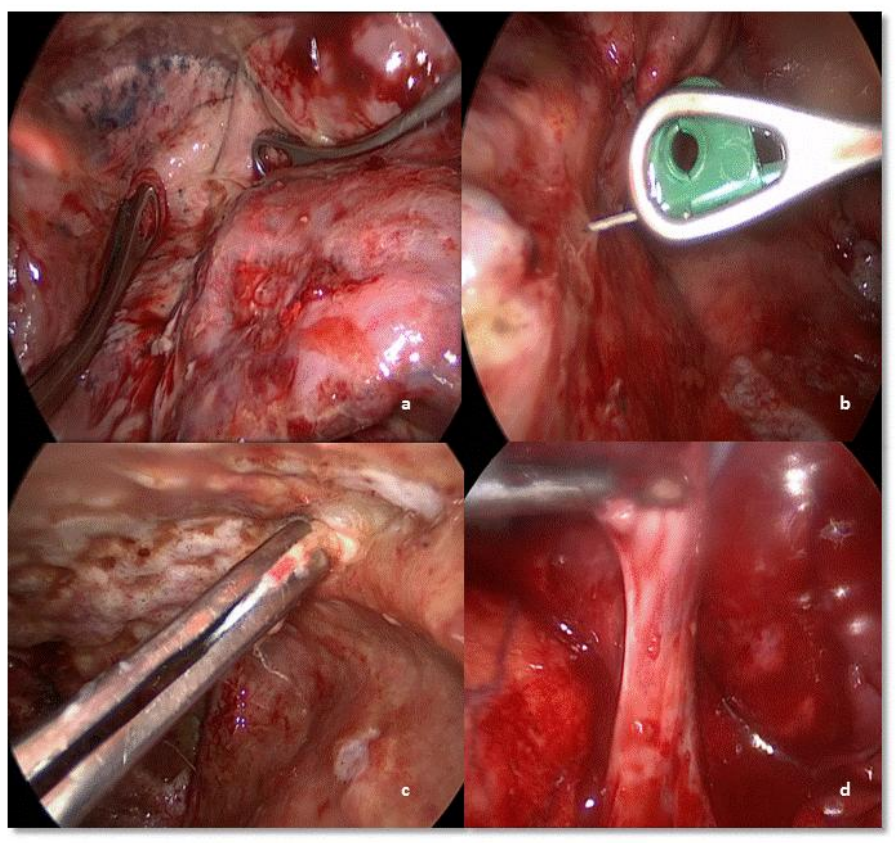

Figura.2- En 2a) Se observa las maniobras de decorticación del lóbulo inferior del pulmón izquierdo liberando lo mayor posible, la cisura mayor; en 2b) se observa la infiltración del nervio frénico para favorecer una parálisis transitoria del hemidiafragma correspondiente; en 2c) mediante maniobras romas se libera las adherencias parietales generadas a nivel del diafragma que favorecen su ascenso en el postoperatorio; en 2d) se muestra e ligamento pulmonar inferior identificado previo a la sección del mismo para permitir mayor ascenso del pulmón homolateral.

Se recolectó para ambos grupos, los datos referentes a la edad, el sexo, el resultado de la anatomía patológica, la demora en la indicación quirúrgica (cuantificándose esta como el número de procedimientos pleurales realizados anteriores a la cirugía), los resultados de la citología pleural obtenidos en la toracocentesis y las complicaciones constatadas durante la internación y los treinta días posteriores a la intervención. Se consideró como complicaciones mayores, a cualquier evento que pudiera relacionarse al procedimiento quirúrgico, que derivó en una re-internación o re-intervención para su resolución, dentro de los 30 días de la cirugía.

El resultado funcional de la pleurodesis, se estimó en base a la mejora en la sensación de disnea, para lo cual se dividió los resultados en mejora buena-regular vs sin mejora para el análisis. La mejora en la disnea se estimó de forma subjetiva durante el seguimiento, mediante preguntas simples relacionadas con el desarrollo de actividades cotidiana (deambulación, vestimenta, realización de tareas simples en el hogar y la persistencia o desaparición de la disnea en reposo). Como parámetro de fracaso de la técnica se tuvo en cuenta la recidiva parcial "sintomática" o la recidiva completa del DPN dentro de los 30 días.

El seguimiento postoperatorio durante la internación, se llevó a cabo con radiografías de tórax cada 48 horas. Sistemáticamente, utilizamos aspiración a $-20 \mathrm{~cm}$ de $\mathrm{H}_{2} \mathrm{O}$, por tres días y el drenaje es retirado cuando el débito es $<150 \mathrm{ml}$ en 24 horas, no existe aerorragia y se constata expansión pulmonar adecuada en la radiografía de tórax. Los controles luego del alta se efectuaron a los 7, 15 y 30 días, con examen físico y control radiológico. Los datos fueron contrastados entre ambos grupos para el análisis.

\section{Análisis estadístico}

Las variables continuas se expresaron como media y desviación estándar o como mediana y rangos en función de su homogeneidad. Las variables categóricas se plasmaron en forma de número y porcentaje. La comparación entre variables se llevó a cabo mediante la prueba de Chi cuadrado de acuerdo a las frecuencias esperadas y la correlación de Pearson, según sus características. Un valor de $\mathrm{p}<0,05$ se consideró significativo. El análisis se llevó a cabo con el software estadístico SPSS 17,0 (SPSS, Inc., Chicago, IL).

\section{Resultados}

Un total de 28 pacientes ingresaron en estudio. Se definieron dos grupos: uno con $11 / 28$ pacientes con DPNC en los que en el $100 \%$ de los casos se realizó alguna maniobra para mejorar la expansión pulmonar, y el grupo de $17 / 28$ pacientes con DPNNC en donde la pleurodesis se realizó sin mayores dificultades. En la tabla 1 se detallan los datos relevantes de la muestra y de cada grupo. La edad promedio fue de 62,64 \pm 12,95 años (rango de 39 - 89 años). La distribución por sexo fue de 22 mujeres $(78,6 \%)$ y 6 hombres $(21,4 \%)$ para toda la muestra. El compromiso pleural por patología mamaria maligna fue lo más frecuente $(46,4 \%)$, seguido del cáncer de pulmón $(21,4 \%)$ y en tercer lugar el mesotelioma maligno $(10,7 \%)$. 
Tabla 1: Datos de las variables en estudio y las diferencias entre los grupos analizados.

\begin{tabular}{|c|c|c|c|c|}
\hline & $n=28$ & $\begin{array}{c}\text { grupo } \\
1 \\
(n=11)\end{array}$ & $\begin{array}{c}\text { grupo } \\
2 \\
(n=17)\end{array}$ & $p$ \\
\hline $\begin{array}{l}\text { Edad (años), } \\
\text { media (SD) }\end{array}$ & $\begin{array}{c}62,64 \\
( \pm 12,9)\end{array}$ & & & \\
\hline $\begin{array}{l}\text { Sexo femenino, } \\
\%\end{array}$ & $\begin{array}{c}22 \\
(78,6 \%)\end{array}$ & & & \\
\hline $\begin{array}{l}\text { Sexo } \\
\text { masculino, \% }\end{array}$ & $\begin{array}{c}6 \\
(21,4 \%)\end{array}$ & & & \\
\hline $\begin{array}{l}\text { Disnea previa a } \\
\text { la pleurodesis, } \\
\%\end{array}$ & $\begin{array}{c}22 \\
(78,6 \%)\end{array}$ & & & \\
\hline $\begin{array}{l}\text { Toracocentesis } \\
\text { previas, (rango) }\end{array}$ & & $\begin{array}{c}2,5 \\
(0-9) \\
\end{array}$ & $\begin{array}{c}2 \\
(0-4) \\
\end{array}$ & \\
\hline $\begin{array}{l}\text { Complicaciones } \\
\text { dentro de los } \\
30 \text { días del } \\
\text { procedimiento } \\
(\%)\end{array}$ & & $\begin{array}{c}7 \\
(63,9 \%)\end{array}$ & $\begin{array}{c}9 \\
(52,9 \%)\end{array}$ & 0,576 \\
\hline $\begin{array}{l}\text { Muertes dentro } \\
\text { de los } 30 \text { días } \\
\text { del } \\
\text { procedimiento } \\
(\%)\end{array}$ & & $\begin{array}{c}3 \\
(27,3 \%)\end{array}$ & $\begin{array}{c}2 \\
(11,8 \%)\end{array}$ & 0,295 \\
\hline $\begin{array}{l}\text { Mejora en la } \\
\text { disnea luego } \\
\text { del } \\
\text { procedimiento } \\
(\%)\end{array}$ & & $\begin{array}{c}3 \\
(81,8 \%)\end{array}$ & $\begin{array}{c}14 \\
(82,4 \%)\end{array}$ & 0,971 \\
\hline $\begin{array}{l}\text { Relación } \\
\text { toracocentesis } \\
\text { - mejora en la } \\
\text { disnea }\end{array}$ & & & & 0,83 \\
\hline $\begin{array}{l}\text { Relación } \\
\text { toracocentesis } \\
\text { - muerte dentro } \\
\text { de los } 30 \text { días }\end{array}$ & & & & 0,008 \\
\hline $\begin{array}{l}\text { Relación } \\
\text { toracocentesis } \\
- \\
\text { complicaciones } \\
\text { dentro de los } \\
30 \text { días }\end{array}$ & & & & 0,002 \\
\hline
\end{tabular}

Tabla 2.- En el grupo con DPNC, en el $81,89 \%$ de los casos el diagnóstico se realizó por biopsia quirúrgica, y solo el $18,18 \%$ de ellos tenía una

\begin{tabular}{ccc} 
Histología & Frecuencia & Porcentaje \\
\hline Cáncer de estomago & 1 & 3,6 \\
\hline Cáncer de mama & 13 & 46,4 \\
\hline Cáncer de pulmón & 6 & 21,4 \\
\hline Linfoma folicular pleural & 1 & 3,6 \\
\hline Mesotelioma pleural & 3 & 10,7 \\
\hline Otras mieloproliferativas & 1 & 3,6 \\
\hline Otros tumores & 3 & 10,8 \\
\hline Total & 28 & 100,0
\end{tabular}

citología pleural positiva para malignidad. En el grupo con DPNNC, la tendencia fue similar con el
$70,59 \%$ de los casos diagnosticados con la cirugía y con $29,41 \%$ con citología pleural

\begin{tabular}{ccc}
\hline & $\begin{array}{c}\text { Grupo A } \\
\text { (DPNC) }\end{array}$ & $\begin{array}{c}\text { Grupo B } \\
\text { (DPNNC) }\end{array}$ \\
\hline Fiebre & $3(27,27 \%)$ & $6(35,29 \%)$ \\
\hline Arritmia SV & $1(9,09 \%)$ & - \\
\hline Hemorragia & $1(9,09 \%)$ & - \\
\hline $\begin{array}{c}\text { Insuf. Respiratoria } \\
\text { Aguda }\end{array}$ & $1(9,09 \%)$ & - \\
\hline Otras complicaciones & $1(9,09 \%)$ & $3(17,76 \%)$ \\
\hline Sin Complicaciones & $4(36,36 \%)$ & $8(47,06 \%)$ \\
\hline Total=n(\%) & $11(100 \%)$ & $17(100 \%)$ \\
\hline positiva. & &
\end{tabular}

Tabla 2: Detalle de los resultados anatomopatológicos de la serie.-

Tabla 3: Complicaciones registradas durante la internación en ambos grupos.

Más del $90 \%$ de los pacientes recibieron más de una toracocentesis como procedimiento previo. La media de punciones fue similar en ambos grupos (2 toracocentesis/paciente), con un rango mayor en el grupo con DPNC (entre $0-9$ vs entre 0 - 4) respectivamente. La estadía hospitalaria fue mayor en los pacientes con DPNC, $(5,81 \pm 0,89$ días) con un mínimo de 3 y un máximo de 11 días para éste grupo. En el grupo sin derrame complicado, los días internación fueron de $3,23 \pm 0,25$ días.

La complicación más frecuente fue la fiebre (temperatura $>38^{\circ} \mathrm{C}$ ) durante la internación $(27,27 \%$ para el grupo con DPNC vs $35,29 \%$ para el grupo con DPNNC). Solo 3 complicaciones mayores asociadas al procedimiento fueron registradas. Un caso de insuficiencia respiratoria aguda con necesidad de ventilación mecánica y un caso de paro cardiorrespiratorio secundario a un evento coronario agudo durante la internación. Por último un paciente en el grupo con DPNC fue reintervenida por sangrado de los puertos de trabajo. La mayoría de procedimientos se llevó a cabo sin eventualidades en ambos grupos. Un total de 5 pacientes $(17,24 \%)$ falleció dentro de los 30 días de seguimiento, dos de ellos mencionados anteriormente y los demás por progresión de la enfermedad de base. No se demostró diferencia entre ambos grupos en relación al índice de complicaciones $(p=0,31)$ y al riesgo de defunción a los 30 días $(p=1,09)$ con la implementación de las maniobras de liberación - expansión o no. Sin embargo, la demora en la indicación quirúrgica estuvo asociada con un mayor índice de complicaciones dentro de los 30 días $(p=0,002)$ y mayor probabilidad de defunción a los 30 días $(\mathrm{p}=0,008)$. 
En cuanto a los resultados funcionales obtenidos luego de la cirugía, el $81,82 \%$ de los pacientes con DPNC y el $82,35 \%$ del grupo con DPNNC evidenciaron una mejora en la disnea, no habiendo diferencia significativa entre ambos grupos. $(p=0,97)$. Casi todos los pacientes tuvieron una reinserción a sus tareas diarias, con buena tolerancia a la disnea luego del procedimiento. No se encontró relación entre los resultados funcionales y la demora en la indicación quirúrgica $(p=0,083)$. Sin embargo el número de toracocentesis arrojó una relación inversa con la el tiempo de internación y la posibilidad de desarrollar un DPNC, con un valor para el coeficiente de correlación de Pearson negativo $(r=-0,34)$.

\section{Discusión}

El desarrollo de un DPN implica una enfermedad avanzada con una sobrevida promedio de 6 meses en la mayoría de los $\operatorname{casos}^{[1,2,3,4]}$. El objetivo en el manejo de estos pacientes terminales es la mejora de su calidad de vida. Por lo tanto, se debe actuar con rapidez evitando el deterioro de la condición clínica ${ }^{[3]}$. No existe un tratamiento estandarizado, sino que debe adecuarse a cada caso en particular ${ }^{[2]}$.

Las guías para el manejo del DPN consideran que en pacientes con falla de expansión pulmonar, la pleurodesis química no es una opción viable. Sin embargo, no brindan mayores detalles sobre los distintos escenarios que pueden presentarse en éste grupo heterogéneo, a la hora de tomar desicione $\mathrm{s}^{[1,5]}$.

En la actualidad, ante la falla de expansión pulmonar, se considera la colocación de catéteres pleurales tunelizados como la opción más razonable ${ }^{[6,7,8]}$. En contraparte, la pleurodesis química con talco por videotoracoscopía se reserva para pacientes con un DPN recidivado, con buena expansión pulmonar y con un buen estado general ${ }^{[9]}$. Sin embargo, aun el paciente ideal, puede cursar con liquido residual en el postoperatorio sin repercusión clínica en su evolución. Por lo que un resultado satisfactorio, no requiere necesariamente que se logre una expansión completa del pulmón.

Con cierta frecuencia, la falta de expansión pulmonar se debe a depósitos de fibrina, coágulos y adherencias laxas entre la superficie pulmonar y la pared torácica constatados mediante la semiología quirúrgica del espacio pleural. La valoración del compromiso pleuropulmonar basado en imágenes, no permite esta evaluación y puede ser causa del descarte de pacientes para un procedimiento de sellado pleural definitivo satisfactorio. Es decir, hay situaciones en las que la expansión pulmonar se ve afectada por causas "potencialmente modificables" y que son pasibles de resolución.

En este trabajo, el sellado pleural con una expansión total o casi total del pulmón afectado se logró en el $81 \%$ de los casos con DPNC, y la decisión de completar el procedimiento solo pudo llevarse a cabo mediante la valoración por visión directa del espacio pleural y la superficie pulmonar. El uso de las "maniobras de liberación - expansión pulmonar" descriptas se implementó si se estimaba que el paciente tenía una expectativa de vida más allá de los 3 meses al momento del procedimiento. Los resultados obtenidos con la pleurodesis química fueron similares para ambos grupos con índices de complicaciones y fracasos similares, con lo cual la instrumentación del espacio pleural parece una opción segura.

Durante el período de internación se constataron dos decesos posiblemente relacionados con el acto quirúrgico. Es de destacar que ambos pacientes registraban el mayor número de toracocentesis realizadas previamente (uno con 4 evacuaciones, el otro 9 evacuaciones previas) de toda la serie. Debido a que es difícil definir la demora en el tratamiento oportuno del DPN, consideramos que cuantificar los procedimientos pleurales previos (toracocentesis) antes de la derivación y la indicación de pleurodesis es un parámetro bastante cercano. Bernard et al. ${ }^{10}$ analizó estas variables y determinó que un mayor número de toracocentesis previas, predice una mayor mortalidad a los 90 días del procedimiento. En nuestro trabajo, cuando analizamos la repercusión de este parámetro en los resultados obtenidos, observamos que aumentaría la posibilidad de cursar con complicaciones o de fallecer dentro de los 30 días posteriores al procedimiento $(p=0,002$ y $\mathrm{p}=0,008$ respectivamente). También aumenta al parecer el tiempo de estadía hospitalaria. En la literatura se describen otros parámetros a tener en cuenta al momento de seleccionar los pacientes para la pleurodesis química por VTC/VATS, como la albúmina sérica $<3,5 \mathrm{~g} / \mathrm{l}$ y el status performance que podrían inferir en los resultados ${ }^{[10,11]}$.

El deterioro clínico repercute en el éxito de un sellado pleural efectivo. Trabajos como los de Steger et al. ${ }^{[12]}$ y Reddy et al. ${ }^{[3]}$ proponen la realización de una pleurodesis temprana, cuando el deterioro del estado general aun es poco evidente. En éstos trabajos el sellado pleural se realizó ante la primera recidiva, alcanzando un éxito en el $92 \%$ de los casos. De la misma forma, consideramos que no existe motivo alguno para 
demorar la indicación quirúrgica en pacientes con una primera recidiva de su derrame pleural o que debutan con el mismo durante el tratamiento oncológico. En estos casos es en dónde se logran los mejores resultados, lográndose una reinserción a las actividades en un breve lapso de tiempo posterior al procedimiento. En esta serie, ningún paciente con una pleurodesis química realizada ante la primera recidiva o luego de su aparición durante el tratamiento oncológico cursó con un DPNC. Así, el 88,24\% de ellos tuvo procedimiento sin complicaciones. La pleurodesis química temprana, también ha sido propuesta por otros autores con buenos resultados, aun cuando el agente utilizado fue la bleomicina como esclerosante ${ }^{[13]}$.

En relación a los resultados funcionales, es probable que a mayor número de procedimientos pleurales previos, existiría una mayor posibilidad de no concretar buenos resultados con la técnica $(p=0,083)$. Este punto, estaría ligado al deterioro progresivo que sufren los pacientes cuando se demora la derivación o la indicación quirúrgica. Parámetros como un índice de Karnofsky > 60 \% y un IMC $>25 \mathrm{~kg} / \mathrm{m} 2$ se asociaron a una mayor sobrevida a los 90 días y pueden ser tenidos en cuenta a la hora de decidir la pleurodesis temprana y la realización de maniobras de liberación para mejorar la expansión pulmonar $^{[2,12,14]}$. Nosotros utilizamos la escala Karnofsky en la evaluación de nuestros pacientes, efectuando las maniobras en aquellos con valores de la escala $>70$.

Los puntos débiles de nuestro trabajo radican en el carácter retrospectivo del mismo, y en el tamaño pequeño de la muestra como para obtener evidencia más sólida. Otro punto a tener presente es, que no se evaluó si las maniobras de liberación aumentan considerablemente el tiempo operatorio, y si esto determina la posibilidad de desarrollar complicaciones en el postoperatorio en éste grupo de pacientes. Tampoco se constató cuál de las maniobras descriptas es más efectiva por sí misma, o si alguna de ellas no tiene valor en su implementación, ya que no se las comparó entre sí, pero se comprobó que la suma de todas ellas llevan a mejorar la expansión pulmonar para un sellado pleural satisfactorio.

Por último, el trabajo no compara el manejo del DPNC mediante VATS/VTC con otras opciones terapéuticas propuestas en el manejo del derrame pleural neoplásico avanzado. En cuanto a si la demora en la indicación de la pleurodesis tiene inferencia en los resultados o no, es difícil de determinar con un grupo tan heterogéneo de pacientes, dado que las comorbilidades asociadas también influyen en el estado general y los resultados al momento de decidir la pleurodesis.

\section{Conclusión}

La falta de expansión pulmonar valorada por imágenes, no implica descartar por completo la cirugía y privar a los pacientes del beneficio del sellado pleural definitivo. En los pacientes con DPNC, las "maniobras de liberación - expansión pulmonar" descriptas, aumentarían las chances de mejorar los resultados en casos seleccionados, sin aumentar la morbilidad del procedimiento propiamente dicho.

La pleurodesis química temprana mejora la calidad de vida de los pacientes portadores de un DPN. La demora en su indicación precoz, aumentaría de forma innecesaria el número de procedimientos pleurales previos no exentos de complicaciones y sin mayores beneficios a largo plazo, y posiblemente también, acortaría la sobrevida en los pacientes con DPN.

Para finalizar, la pleurodesis química temprana es una opción a tener presente en el manejo de pacientes con DPN con escasa accesibilidad al sistema de salud, como ocurre en distintas regiones de nuestro país, en donde la implementación otras opciones terapéuticas y el seguimiento por parte del sistema de salud son complicados.

\section{Bibliografía}

1. Roberts ME, Neville E, Berrisford RG, Antunes G, Ali NJ, on behalf of the BTS Pleural Disease Guideline Group. Management of the malignant pleural effusion: British Thoracic Society pleural disease guideline 2010. Thorax. 2010; 65(Suppl 2):32-40. [PUBMED]

2. Haas AR, Sterman DH, Musani Al. Malignant Pleural Effusions: Management options with consideration of coding, billing, and a decision approach. Chest. 2007; 132:1036-41. [PUBMED]

3. Reddy Ch, Ernst A, Lamb C, Feller-Kopman D. Rapid Pleurodesis for Malignant Pleural Effusions: A Pilot Study. Chest. 2011; 139:1419-23. [PUBMED]

4. Swiderek J, Morcos S, Donthireddy V, Surapaneni R, Jackson-Thompson $V$, Schultz $L$, et al. Prospective study to determine the volume of pleural fluid required to diagnose malignancy. Chest. 2010; 137:68-73. [PUBMED]

5. Villena Garrido V, Ferrer Sancho J, Hernández Blasco L, De Pablo Gafas A, Pérez Rodríguez E, Rodríguez Panadero $F$, et al. Área de Técnicas y Trasplante. SEPAR. Diagnóstico y tratamiento del derrame pleural. Arch Bronconeumol. 2006; 42:349-72. [PUBMED]

6. Light RW. Counterpoint: Should thoracoscopic talc pleurodesis be the first choice management for malignant pleural effusion? No. Chest 2012;142:17-19. [PUBMED]

7. Hunt BM, Farivar AS, Vallières E, Louie BE, Aye RW, Flores EE, et al. Thoracoscopic talc versus tunneled pleural catheters for palliation of malignant pleural effusions. Ann Thorac Surg. 2012; 94:1053-9. [PUBMED] 
8. Davies HE, Mishra EK, Kahan BC, Wrightson JM, Stanton $A E$, Guhan $A$, et al. Effect of an indwelling pleural catheter vs chest tube and talc pleurodesis for relieving dyspnea in patients with malignant pleural effusion. The TIME2 randomized controlled trial. JAMA. 2012; 307:2383-89. [PUBMED]

9. Tan C, Sedrakyan A, Browne J, Swift S, Treasure T. The evidence on the effectiveness of management for malignant pleural effusion: a systematic review. Eur $J$ Cardiothoracic Surg. 2006; 29:829-38. [PUBMED]

10. Bernard A, Bernard de Dompsure R, Hagry O, Favre JP. Early and late mortality after pleurodesis for malignant pleural effusion. Ann Thorac Surg. 2002; 74:213-7. [PUBMED]

11. Pilling JE, Dusmet ME, Ladas $G$, Goldstraw $P$. Prognostic factors for survival after surgical palliation of malignant pleural effusion. J Thorac Oncol. 2010; 5:1544-50. [PUBMED]

12. Steger V, Mika U, Toomes $H$, Walker T, Engel $C$, Kyriss $T$, et al. Who gains most? A 10-year experience with 611 thoracoscopic talc pleurodeses. Ann Thorac Surg. 2007; 83:1940-5. [PUBMED]

13. Spiegler PA, Hurewitz AN, Groth ML. Rapid pleurodesis for malignant pleural effusions. Chest. 2003; 123:1895-8. [PUBMED]

14.Dresler CM, Olak J, Herndon II JE, Richards WG, Scalzetti E, Fleishman SB, et al. Phase III intergroup study of talc poudrage vs talc slurry sclerosis for malignant pleural effusion. Chest 2005; 127:909-15. [PUBMED] 